\title{
INOCULACIÓN CON HONGOS MICORRÍZICOS Y FERTILIZACIÓN CON UREA DE PLANTAS DE FRAXINUS UHDEI EN ACRISOLES PROVENIENTES DE SITIOS DEGRADADOS
}

\author{
Ana Laura Báez-Pérez ${ }^{1}$, Mariela Gómez-Romero², Javier VillegGas', Erick de la Barrera³ \\ Lorena Carreto-Montoya ${ }^{1}$ y Roberto Lindig-Cisneros ${ }^{3,4}$ \\ 'Instituto de Investigaciones Químico-Biológicas, Universidad Michoacana de San Nicolas de Hidalgo. \\ ${ }^{2}$ Escuela Nacional de Estudios Superiores (ENES) Unidad Morelia. Universidad Nacional Autónoma de México, \\ Morelia, Michoacán, México. \\ ${ }^{3}$ Laboratorio de Ecología de Restauración, Centro de Investigaciones en Ecosistemas, \\ Universidad Nacional Autónoma de México. Campus Morelia, Michoacán, México. \\ ${ }^{4}$ Autor para la correspondencia: rlindig@cieco.unam.mx.
}

\begin{abstract}
Resumen: En suelos severamente degradados el establecimiento de plantas se dificulta por la ausencia de estructura, de microorganismos del suelo y en muchos casos por las bajas concentraciones de macronutrientes. En estas condiciones, el uso de interacciones con hongos micorrízicos, podría ser una estrategia efectiva para la revegetación y la restauración ecológica. Se realizó un experimento con sustrato obtenido de un sitio severamente erosionado (acrisoles con presencia de cárcavas) en un sistema de mesocosmos, con el objetivo de evaluar el desempeño de Fraxinus uhdei (Wenz.) Lingelsh (fresno) inoculado con los hongos micorrízicos Pisolithus tinctorius y Rhizophagus intraradices, solos o en interacción y en presencia/ausencia de fertilización nitrogenada. Se evaluó la altura, la cobertura de la copa, el diámetro a la altura de la base del tallo y el número de hojas al finalizar el experimento y se cosechó la biomasa. Se prepararon muestras para verificar la micorrización. Los resultados indicaron que la fertilización no tuvo efecto significativo sobre el desarrollo de las plantas pero, afectó negativamente la supervivencia (con urea $67 \%$, sin urea $90 \%$ ). Los mayores valores para las variables de crecimiento y la biomasa aérea siempre correspondieron a las plantas con inoculación dual, y los menores para las plantas control o las inoculadas con P. tinctorius (altura $23.3 \mathrm{~cm}$ contra $15.8 \mathrm{~cm}$ para $P$. tinctorius, diámetro a la altura de la base $0.74 \mathrm{~cm}$ contra 0.5 para $P$. tinctorius, el peso seco de las partes aéreas $2.0 \mathrm{~g}$ contra $1.0 \mathrm{~g}$ del control). Los resultados sugieren que en suelos que presentan condiciones severas de degradación la co-inoculación de plantas adaptadas a condiciones adversas puede ser una estrategia eficiente de revegetación
\end{abstract}

Palabras clave: co-inoculación, Pisolithus tinctorius, Rhizophagus intraradices, restauración ecológica, simbiosis.

\begin{abstract}
In severely degraded soils, plant establishment is difficult because of lack of structure, a depleted community of microorganisms and in many sites low concentrations of macronutrients. Under these conditions, the use of interactions with mycorrhizal fungi could be an effective strategy for revegetation and ecological restoration. An experiment was established in mesocosms with soil gathered from a severely degraded site (acrisols with gully formations). The objective of the study was the evaluation of the performance of Fraxinus uhdei (Wenz.) Lingelsh (ash) inoculated with the mycorrhizal fungi Pisolithus tinctorius and Rhizophagus intraradices, each species by itself or in combination and also with presence or absence of nitrogen fertilization. Height, canopy cover, diameter at the base of the stem and number of leaves were evaluated at the end of the experiment, afterwards biomass was harvested. Samples were prepared to verify mycorrhization. Results showed that fertilization had no significant effect on plant development but had a negative effect on survival (with urea $67 \%$, without urea $90 \%$ ). The highest values for growth variables and biomass were those of plants with dual inoculation, and the lowest for control plants or plants inoculated with P. tinctorius (height $23.3 \mathrm{~cm}$ against $15.8 \mathrm{~cm}, \mathrm{DAB} 0.74 \mathrm{~cm}$ against 0.5 , dry aboveground weight $2.0 \mathrm{~g}$ against $1.0 \mathrm{~g}$ ). These results suggest that in severely degraded soils dual inoculation of plant species tolerant to degraded sites can be an efficient revegetation strategy.
\end{abstract}

Key words: co-inoculation, ecological restoration, Pisolithus tinctorius, Rhizophagus intraradices, symbiosis.

U no de los problemas ambientales más graves y mundialmente reconocidos es la erosión del suelo, resultado principalmente de la deforestación. En México, la tasa de deforestación alcanzó hace 20 años cerca de 800,000 ha/año
(Masera et al., 1992), y se estima que se perdió más suelo en la segunda mitad del siglo XX que en los cuatro siglos anteriores (Maass y García-Oliva, 1990). La erosión recurrente de la capa superficial del suelo, y la erosión en cárcavas 
como consecuencia de la deforestación y técnicas de cultivo inadecuadas, han sido documentadas para el 65 o el $85 \%$ de la superficie de México (Bocco y García-Oliva, 1992; Maass y García-Oliva, 1990), lo anterior implica que cerca de 535 millones de toneladas de suelo se pierden anualmente (SEMARNAT, 1997).

La lluvia, el viento, el tipo de suelo, la pendiente, la cobertura vegetal y la presencia o ausencia de medidas de conservación son los factores principales que determinan la erosión del suelo (Pimentel, 2006). Debido a que en los suelos altamente erosionados no existe una cubierta vegetal que brinde protección, la lluvia cae directamente, provocando con ello que el flujo de agua de lluvia empiece a arrastrar el suelo provocando la erosión por cárcavas, un grado avanzado de erosión (Miller, 1994). En suelos con alto grado de erosión, se altera o pierde considerablemente la diversidad de microbiota (Nie et al., 2013), se reduce la capacidad de retención de la humedad, se modifica la estructura, el nivel de compactación, se puede dar el afloramiento de subhorizontes con menor fertilidad, lo que en su conjunto se traduce eventualmente en la pérdida total del suelo y en la presencia de un sustrato sin las propiedades físicas y químicas mínimas para el desarrollo y supervivencia de las plantas (Durán y Rodríguez, 2008).

En suelos altamente erosionados, para evitar mayores daños en el sitio y en las cuencas en los que se encuentran, es recomendable establecer programas de restauración que entre otras medidas deben contemplar el establecimiento de una cobertura vegetal para mejorar la estructura de suelo y por lo tanto facilitar su regeneración y protección. En ese sentido el estudio de plantas con potencial para formar asociaciones simbióticas tanto con hongos micorrízicos arbusculares (HMA) como con hongos ectomicorrízicos (HE), puede contribuir a mejorar las estrategias de restauración ecológica (Gómez-Romero et al., 2012), en particular lo relacionado con la diversidad microbiana del suelo y las funciones que esta diversidad sostiene.

Una función bien documentada de los hongos micorrízicos es la de facilitar a la planta la adquisición y la absorción de fósforo. Sin embargo, estos hongos procuran otros beneficios a las plantas, tanto a escala poblacional como de comunidades: mejoran la adquisición y la absorción del nitrógeno y del cobre, limitan la absorción de metales pesados tóxicos como el zinc y el cadmio. Además, mejoran el flujo de agua, aumentando la resistencia de las plantas a la sequía, particularmente en las primeras etapas de vida. También incrementan la resistencia contra agentes patógenos y, por consiguiente, aumentan la adecuación de las plantas, su supervivencia y su fecundidad (Smith y Read, 1997; PérezMoreno y Read, 2004).

Las especies vegetales que forman asociaciones micorrízicas presentan una fisiología y una ecología diferentes de aquellas que no forman esta asociación, y se considera a la asociación micorrízica como uno de los factores promotores de la diversidad vegetal, al facilitar el establecimiento de las plantas (Pohl et al., 2009; Smith et al., 2010).

Unas de las especies vegetales capaces de establecerse en sitios degradados son los fresnos (Fraxinus), en particular Fraxinus uhdei, pues son resistentes a suelos pobres en nutrientes y materia orgánica (Francis, 1990; Stabler et al., 2001). Fraxinus uhdei es una especie endémica a México capaz de formar asociaciones simbióticas en condiciones de laboratorio (Ambríz et al., 2010) tanto con hongos ectomicorrízicos (HEM) como con hongos endomicorrízicos (HMA). Aunado a ello, Rothstein et al. (2004), documentaron que hay grandes cantidades de $\mathrm{N}$ y $\mathrm{P}$ en la hojarasca de un bosque dominado por fresnos, y por ende plantean que esta especie favorece la recuperación de la dinámica biogeoquímica del suelo.

Por otro lado, se ha sugerido que el éxito del fresno para poder desarrollarse en zonas degradas puede ser el resultado de su habilidad para formar asociaciones simbióticas con hongos micorrízicos (Stabler et al., 2001). Sin embargo, existen pocos trabajos que muestran la contribución del sistema fresno-hongos micorrízicos en el desempeño de la planta en sustratos limitantes.

Las variables que afectan esta asociación bajo condiciones más realistas pero aun controladas no han sido estudiadas. Lo anterior se puede lograr con el uso de mesocosmos y que han probado ser una aproximación experimental útil para la restauración ecológica (Callaway et al., 1997; Szanser et al., 2011). Por lo tanto, el objetivo de este estudio fue evaluar el desempeño de Fraxinus uhdei inoculado con los hongos micorrízicos Pisolithus tinctorius (Pers.) Coker \& Couch y Rhizophagus intraradices (N.C.Schenck \& G.S.Sm.) C.Walker \& A.Schüßler (syn. Glomus intraradices; Schüßler y Walker 2010; Krüger et al., 2012), solos o en interacción en un experimento de mesocosmos, a fin de evaluar el comportamiento del sistema en un suelo con las características antes mencionadas, partiendo de la hipótesis de que la adición de $\mathrm{N}$ incrementará el desempeño y supervivencia del sistema $F$. uhdei-R. intraradices-P. tinctorius.

\section{Materiales y métodos}

Se propagaron plantas de Fraxinus uhdei en agosto de 2011 en condiciones de casa de sombra (20\%), para lo cual se escarificaron semillas de forma manual y se desinfectaron con peróxido de hidrógeno $\left(\mathrm{H}_{2} \mathrm{O}_{2}\right)$ al $10 \%$ (v:v) por 15 min. Posteriormente fueron sembradas en contenedores rígidos de plástico $\left(240 \mathrm{~cm}^{3}\right)$, utilizando como sustrato agrolitaturba $(2: 1 \mathrm{v} / \mathrm{v})$ esterilizada en autoclave por 30 minutos a $121^{\circ} \mathrm{C}$ y 15 PSI. A las 9 semanas de crecimiento, las plantas fueron separadas cuatro grupos, las del primer grupo no fueron inoculadas y las pertenecientes a los tres restantes fueron inoculadas: (a) el hongo ectomicorrízico Pisolithus tinctorius $\left(1 \times 10^{6}\right.$ esporas/planta) utilizando como vehículo turba micronizada, (b) el hongo micorrízico arbuscular Rhi- 
zophagus intraradices (50 esporas/planta), aplicado como una suspensión en agua destilada y c) con una mezcla de ambas especies de hongos (inoculación dual), cada especie aplicada como ya se mencionó. El inóculo de P. tinctorius fue obtenido de Biosyneterra Solutions Inc. (L'Assomption, Québec, Canada), y R. intraradices a partir de cultivos aislados en el Laboratorio de Interacción Suelo-Planta-Microorganismo del Instituto de Investigaciones Químico Biológicas de la Universidad Michoacana de San Nicolás de Hidalgo.

Cuando las plantas de fresno cumplieron 17 semanas de inoculación, fueron trasplantadas a contenedores de plástico de 201 que contenían como sustrato suelo extraido de la localidad Huertitas, en el Ejido de Atécuaro (19 33' 05' y $19^{\circ} 37^{\prime} 08^{\prime \prime} \mathrm{N}$ y $101^{\circ} 05^{\prime} 07^{\prime \prime} \mathrm{O}$ y altitud de $2,275 \mathrm{~m}$ ) localizado a $15 \mathrm{~km}$ al sur de la ciudad de Morelia, Michoacán. El suelo no fue esterilizado porque el experimento buscaba lograr condiciones similares a las que las plantas encontrarían en campo pero controlando el mayor número de variables posibles. No esterilizar el sustrato potencialmente permitiría a las plantas asociarse con bacterias presentes en el mismo aunque podría también implicar que las plantas adquirieran otras especies de hongos micorrízicos. Esta localidad se caracteriza por tener un alto grado de erosión y numerosas cárcavas, formadas en suelos de tipo acrisol ócrico (Duvert et al., 2010). En las condiciones actuales de degradación, el suelo de la localidad contiene muy poca materia orgánica $(0.78 \%)$, es muy bajo en nitrógeno total aprovechable $(19.60 \mathrm{~kg} / \mathrm{Ha})$ y extremadamente pobre en fósforo asimilable $(0.347 \mathrm{mg} / \mathrm{kg})$. El experimento fue establecido en el Centro de Investigaciones en Ecosistemas de la Universidad Nacional Autónoma de México, Campus Morelia, al aire libre. Las macetas fueron distribuidas en un diseño completamente al azar con ocho tratamientos (cuatro de inoculación y dos de fertilización en un diseño ortogonal) y 18 réplicas conteniendo una planta de fresno por maceta. El experimento fue evaluado durante 20 meses.

Para los tratamientos con fertilización, urea $\left(\mathrm{NH}_{2} \mathrm{CONH}_{2}\right)$ fue disuelta en agua a una concentración $200 \mathrm{mM}$, de tal forma que cada planta recibió $0.096 \mathrm{~g}$ de urea, cada tercer día durante la temporada de lluvias. El riego se mantuvo constante cada tercer día para todos los tratamientos durante todo el experimento, con excepción de la época de lluvias en la que se suspendió el riego.

Para evaluar el desempeño de los fresnos, se registró al finalizar el experimento la supervivencia, la altura, el diámetro a la altura de la base (DAB en el cuello de la planta a un $\mathrm{cm}$ del suelo), la cobertura (evaluado mediante la fórmula de la elipse $r_{1} r_{2} \pi$ con dos radios de la copa para inferir el dosel), el número de hojas. Se cosechó la biomasa aérea y de raíces de diez plantas por tratamiento elegidas al azar. A partir de este material se obtuvo el peso freso y seco, y para las raíces se midió la longitud, se obtuvo el volumen, por el método de desplazamiento de agua.
El hongo micorrizico arbuscular se identificó por medio de la técnica de tinción tinta y vinagre descrita por Vierheilig et al. (1998), clareando las raíces con $\mathrm{KOH}$ al $10 \%$ (peso/vol) por $30 \mathrm{~min}$, para posteriormente teñir con tinta shaeffer Negra disuelta al $10 \%$ con ácido acético al $25 \%$, y posteriormente lavando el exceso de colorante con agua corriente. Una vez teñidas las raíces se buscaron estructuras características propias de la especie (arbúsculos, vesículas y/o micelio) fotografiando con una cámara Leica DFC 295 (Versión 7.0.1.0) la cual está integrada a un software especializado (Leica Application Suite Versión 3.4.1). Pisolithus tinctorius se detectó en base a la técnica de Thomson $e t$ al. (1993), tiñendo el manto y el micelio externo, del hongo asociado a los segmentos finos de raíces de fresno, con azul de tripano al $0.05 \%$ por 24 horas y retirándose posteriormente el exceso de colorante con agua destilada, antes de ser observados al microscopio.

Los análisis estadísticos se llevaron a cabo a través de análisis de varianza de dos vías, inoculación y fertilización, y se verificó que se cumpliera con los supuestos de este método estadístico. Debido a la mortalidad, y debido a la sensibilidad del análisis de varianza de más de una vía a datos desbalanceados, se eliminaron réplicas al azar de tal forma que se contara con el mismo número de réplicas por tratamientos (Underwood, 1997). En el caso de que para alguna de las variables uno de los factores no fuera significativo, el factor fue eliminado del análisis, lo que permitió en estos casos utilizar la base de datos completa (Crawley, 2007). Todos los análisis se llevaron a cabo con el paquete $\mathrm{R}(\mathrm{R}$ Development Core Team, 2013).

\section{Resultados}

Las preparaciones de tejido de raíz mostraron que la inoculación con Pisolithus tinctorius y Rhizophagus intraradices fue efectiva (Figura 1), tanto para las plantas inoculadas con cada una de estas especies como para aquellas que fueron inoculadas por ambas. Al finalizar el experimento, a los 20 meses de establecido, la mortalidad entre los individuos asignados a los diferentes tratamientos difirió. Entre tratamientos de inoculación las diferencias no fueron significativas $\left(\chi^{2}=2.72\right.$, g.l. $\left.=3, P=0.43\right)$, pero entre tratamientos de fertilización si $\left(\chi^{2}=12.64\right.$, g.1. $\left.=1, P=0.0004\right)$, debido a que la supervivencia sin aplicación de urea fue del $90 \%$ y con aplicación de $67 \%$.

Las variables de crecimiento mostraron, al igual que todas las variables de respuesta consideradas, sólo diferencias entre tratamientos de inoculación. La altura (Figura 2A), fue mayor para las plantas con inoculación dual (GP), que alcanzaron una altura de $23.3 \pm 6.9 \mathrm{~cm}$, y la menor fue para las plantas inoculadas con Pisolithus tinctorius (P), que alcanzaron una altura de $15.8 \pm 4.4 \mathrm{~cm}$, las diferencias entre tratamientos fueron significativas $\left(F_{(3,109)}=7.15, P=\right.$ 0.0002). El diámetro a la altura de la base (DAB) mostró un 


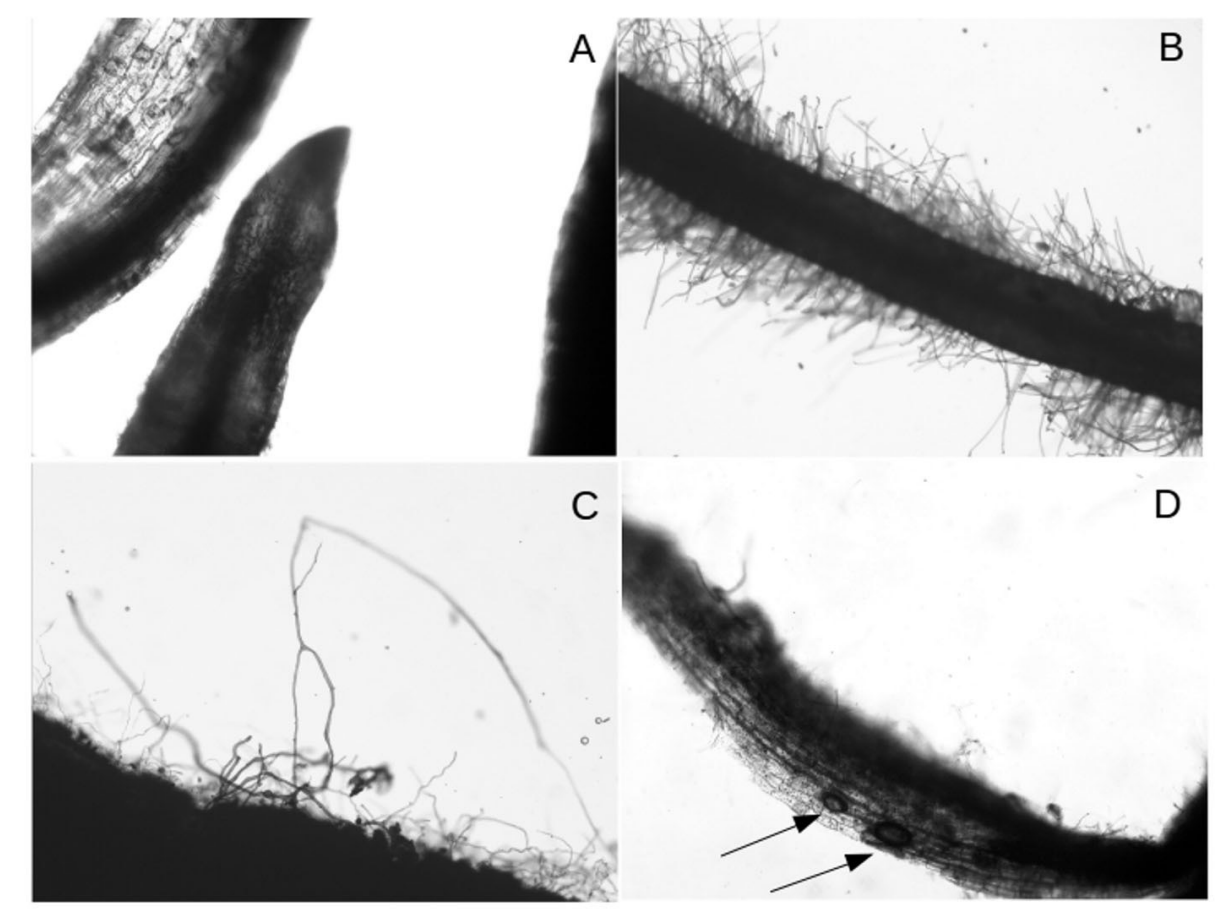

Figura 1. En A) raíces de Fraxinus uhdei pertenecientes al tratamiento control; en B) raíces de la misma especie con micelio externo de Pisolithus tinctorius; en C) raíces con micelio externo de Rhizophagus intraradices y en D) vesículas de Rhizophagus intraradices (indicadas por las flechas).

patrón similar, el mayor diámetro lo presentaron las plantas con inoculación dual, $0.74 \pm 0.27 \mathrm{~cm}$ y el menor las plantas inoculadas con $P$. tinctorius, $0.50 \pm 0.12 \mathrm{~cm}$, las diferencias entre tratamientos fueron significativas $\left(F_{(3,109)}=5.43, P=\right.$ 0.002). La cobertura mostró un patrón similar al de las dos variables anteriores pero en este caso las diferencias se dieron entre las plantas con inoculación dual, las que fueron inoculadas con $P$. tinctorius y las que pertenecían al grupo control (Figura 2C), con coberturas de $88.9 \pm 74.7 \mathrm{~cm}^{2}, 21.2$ \pm 33.1 y $45.6 \pm 57.9 \mathrm{~cm}^{2}$, respectivamente $\left(F_{(3,109)}=5.3, P=\right.$ $0.002)$. Finalmente, para este tipo de variables, el número de hojas siguió el mismo patrón que la cobertura, con el mayor número de hojas para la inoculación dual y la menor para las plantas inoculadas con P. tinctorius y las plantas control, 9.6 $\pm 5.9,6.3 \pm 3.6$ y $6.5 \pm 3.5$ hojas respectivamente $\left(F_{(3,109)}=\right.$ $3.42, P=0.02$ ).

La biomasa de las partes aéreas fue mayor para las plantas con inoculación dual (Figura 3), la biomasa fresca alcanzó $4.77 \pm 3.4 \mathrm{~g}$ para estas plantas en contraste con $2.3 \pm$ $2.2 \mathrm{~g}$ para las plantas control $\left(F_{(3,74)}=4.85, P=0.004\right)$, la biomasa seca siguió el mismo patrón entre tratamientos, las plantas con inoculación dual alcanzaron un peso promedio de $2.0 \pm 1.42 \mathrm{~g}$ contra $1.0 \pm 0.99 \mathrm{~g}$ de las plantas control $\left(\mathrm{F}_{(3)}\right.$. 74) $=4.42, P=0.006$ ).

El peso fresco y seco de las raíces (Figura 4A B) muestran nuevamente un patrón en donde los valores mayores son para las plantas con inoculación dual (peso fresco $=13.9 \pm$ $9.6 \mathrm{~g}$, peso seco $=3.3 \pm 2.6 \mathrm{~g}$ ) y los menores para las plantas inoculadas con Pisolithus tinctorius (peso fresco $=4.4 \pm 3.6$ $\mathrm{g}$, peso seco $=1.0 \pm 0.8 \mathrm{~g}$ ), que no difirieron estadísticamente de los valores para las plantas control (peso fresco $=5.1$ $\pm 4.1 \mathrm{~g}$, peso seco $=1.1 \pm 0.9 \mathrm{~g}$ ); las diferencias tanto para el peso fresco $\left(F_{(3.74)}=7.19, P=0.0003\right)$, como para el peso $\operatorname{seco}\left(F_{(3,74)}=6.29, P=0.0007\right)$, fueron significativas. El volumen de las raíces (Figura 4C) mostró el mismo patrón pues las plantas con inoculación dual tuvieron un volumen de $13.6 \pm 8.8 \mathrm{~cm}^{3}$, y las plantas inoculadas con P. tinctorius y las control de $4.5 \pm 3.7 \mathrm{~cm}^{3}$ y $5.3 \pm 3.2 \mathrm{~cm}^{3}$ respectivamente $\left(F_{(3,74)}=7.68, P=0.0002\right)$. La longitud específica (Figura 4d) fue menor para las plantas con inoculación dual $\left(35.1 \pm 26.7 \mathrm{~cm} \mathrm{~g}^{-1}\right)$ que en las plantas control $(104.7 \pm 89.4$ $\left.\mathrm{cm} \mathrm{g}^{-1}\right)$, que representaron los extremos para esta variable que fue la última en mostrar diferencias significativas entre tratamientos $\left(F_{(3,74)}=4.66, P=0.005\right)$.

\section{Discusión}

En suelos degradados que son deficientes en nutrientes, carentes de estructura y depauperados en diversidad de microorganismos, puede ser útil el uso de plantas resistentes a situaciones adversas y de aprovechar asociaciones simbióticas con hongos micorrízicos (Smith et al., 2010; GómezRomero et al., 2012). En este sentido, en el presente trabajo se estableció un ensayo en suelo de tipo acrisol ócrico, con deficiencias nutricionales que dificultan el desarrollo de las plantas. En el suelo utilizado, el fósforo se encuentra unido 

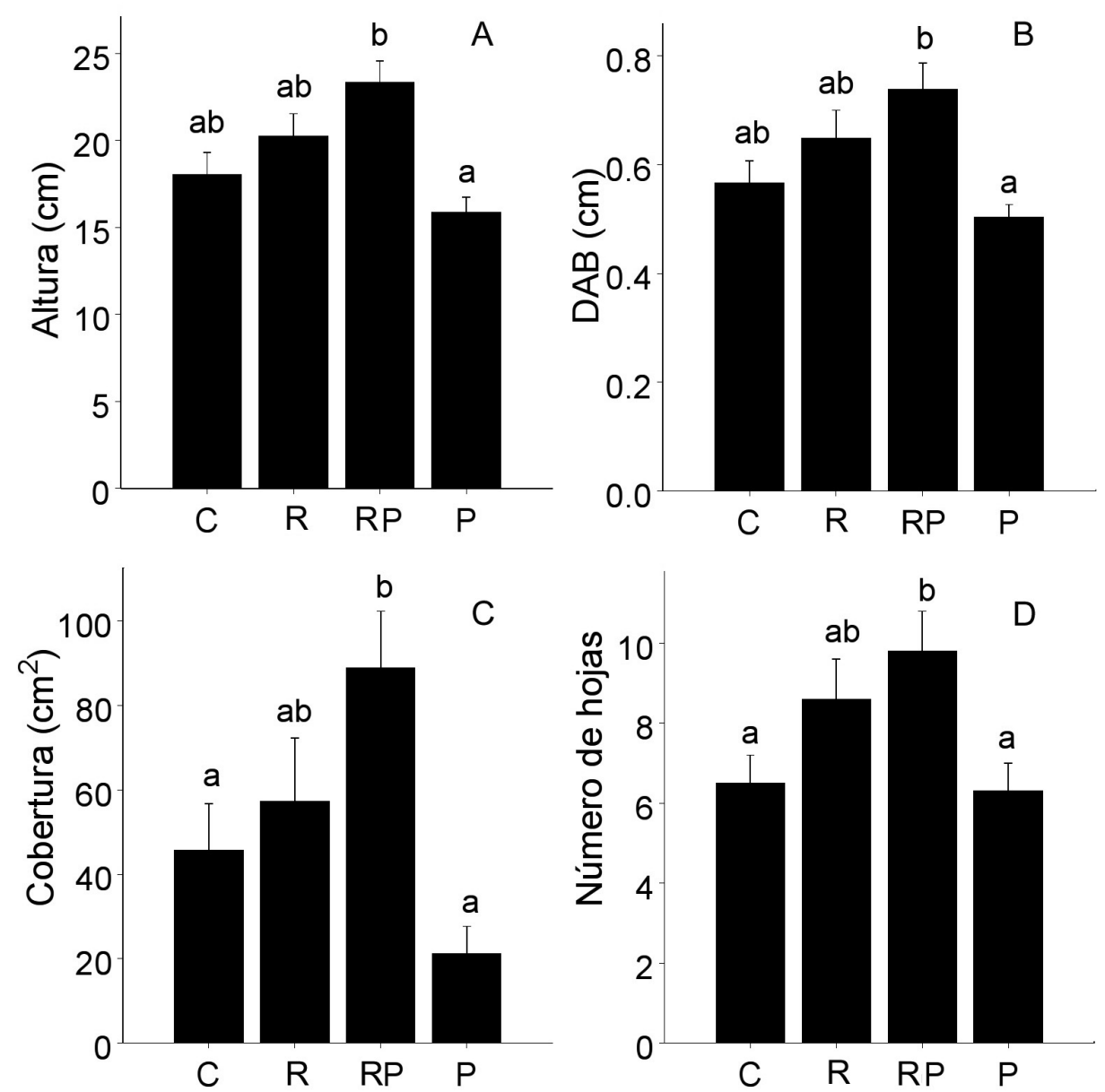

Figura 2. Altura A), diámetro a la altura de la base B), cobertura C) y número de hojas D) de Fraxinus uhdei en los distintos tratamientos de inoculación $(\mathrm{C}=$ control, $\mathrm{R}=$ Rhizophagus intraradices, $\mathrm{P}=$ Pisolithus tinctorius, $\mathrm{GP}=$ inoculación dual). Los datos son medias y desviaciones estándar, las medias no identificadas con la misma letra son significativamente diferentes $(P \leq 0.05)$.

a minerales de hierro, lo que lo hace insoluble y poco disponible para las plantas (ISRIC, 2013). Sin embargo, a pesar de estas características del sustrato se observó que Fraxinus uhdei es capaz de establecerse satisfactoriamente, particularmente si se encuentra formando asociaciones micorrízicas como lo indican los resultados del presente estudio. El
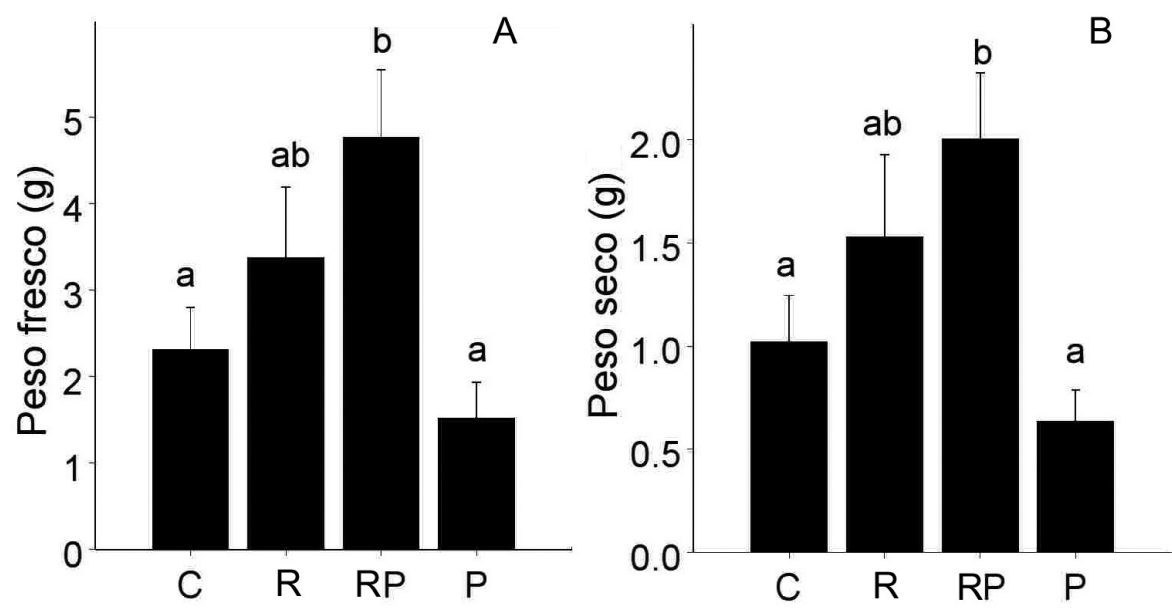

Figura 3. Peso fresco A) y peso seco B) de las partes aéreas de los individuos de Fraxinus udhei cosechados de los diferentes tratamientos de inoculación $(\mathrm{C}=$ control, $\mathrm{R}=$ Rhizophagus intraradices, $\mathrm{P}=$ Pisolithus tinctorius, $\mathrm{GP}=$ inoculación dual $)$. Los datos son medias y desviaciones estándar, las medias no identificadas con la misma letra son significativamente diferentes $(P \leq 0.05)$. 

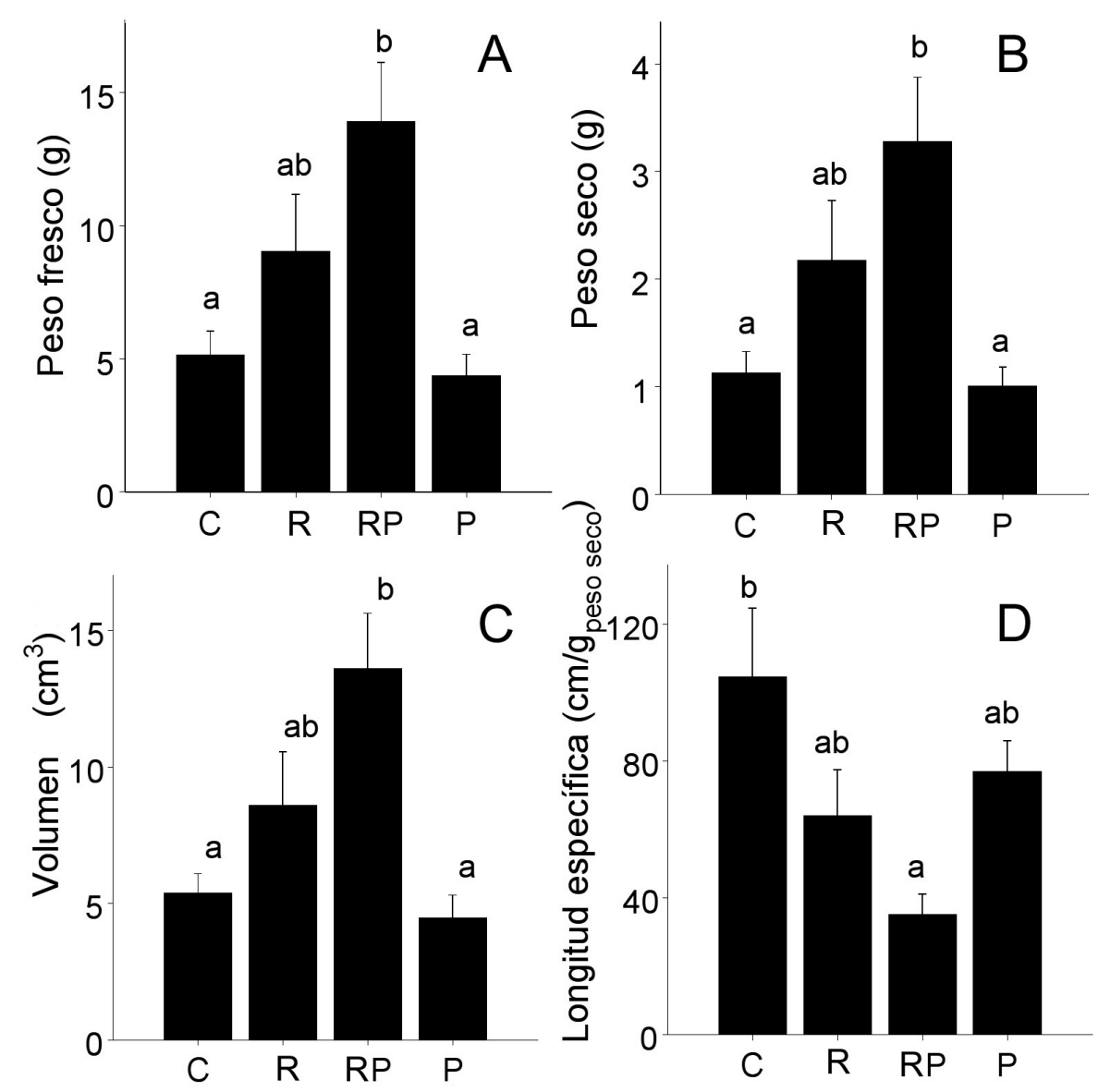

Figura 4. Peso fresco A), peso seco B), volumen C) y longitud específica D) de las raíces de F. uhdei cosechadas de los distintos tratamientos de inoculación $(\mathrm{C}=$ control, $\mathrm{R}=$ Rhizophagus intraradices, $\mathrm{P}=$ Pisolithus tinctorius, $\mathrm{GP}=$ inoculación dual). Los datos son medias y desviaciones estándar, las medias no identificadas con la misma letra son significativamente diferentes $(P \leq 0.05)$.

ensayo mostró que el fresno es resistentes a condiciones adversas del sustrato y sugiere que puede sobrevivir en suelos pobres en nutrientes, aún más pobres que los reportados en otros estudios (Francis, 1990; Stabler et al., 2001).

Es interesante notar que el efecto de la adición de urea fue adverso para la supervivencia de las plantas de fresno en las condiciones experimentales del presente trabajo, y que no tuvo ningún efecto para el resto de las variables evaluadas. Existen pocos trabajos que documenten los efectos de la fertilización nitrogenada en especies arbóreas, y con los que se cuenta, son principalmente de especies del género Pinus. Por ejemplo, para $P$. pinea L., la fertilización no afecta la supervivencia pero si su crecimiento (Cañellas et al., 1999), resultados que son contrarios a lo obtenido en el presente ensayo, donde la supervivencia si se vio afectada pero no así el crecimiento de las plantas, aunque en este sentido los resultados son similares a los obtenidos por Davel et al. (2001), donde se observó que la fertilización nitrogenada no tuvo efecto alguno sobre el desarrollo de P. ponderosa. La micorrización puede verse afectada por la adición de nitrógeno, variando la proporción de las micorrizas presentes, tanto en tipos como en abundancia relativa (Alexander y
Fairley, 1983), de hecho, la adición de nitrógeno inhibe la colonización de los hongos micorrízicos en algunas especies de plantas cultivadas, siendo su efecto depresivo mayor que el del fósforo (Ocampo y Hayman ,1981; Hayman, 1983).

La micorrización tuvo un efecto en todas las variables evaluadas, en particular la micorrización dual, pero no en la supervivencia, lo que contrasta con otros trabajos recientes realizados con leguminosas en zonas semiáridas (MonroyAta et al., 2007), Cedrela odorata (Méndez-Cortés et al., 2013) y Pinus halepensis (Álvarez-Sanchez et al., 2013).

Se sabe que la simbiosis micorrízica estimula el crecimiento vegetal debido principalmente al efecto benéfico sobre la nutrición mineral y el aporte de agua hacia las plantas (Simard y Durall, 2004; Pringle et al., 2009), sin embargo, debe tenerse en cuenta que hay asociaciones distintas que se establecen entre el hongo y su hospedante, y que cada combinación puede tener efectos distintos sobre el crecimiento de la planta. En ese sentido, en este estudio se observó que a pesar de que Fraxinus udhei puede formar asociaciones simbióticas tanto con Rhizophagus intraradices (endomicorriza) como con Pisolithus tinctorius (ectomicorriza), las plantas respondieron de manera distinta a cada especie. Las 
plantas inoculadas con $R$. intraradices tuvieron un mejor crecimiento en comparación con las inoculadas con P. tinctorius y cuando ambos hongos micorrízicos se encuentran en coinoculación se presenta una sinergia que repercute positivamente en el crecimiento de $F$. uhdei. Estos resultados coinciden con lo reportado por Ambriz et al. (2010) para la misma especie de hospedero, sin embargo, el ensayo desarrollado por este autor se realizó bajo condiciones controladas en cámara de crecimiento y en periodo menor de tiempo (12 semanas de duración).

Existen reportes que muestran el efecto benéfico de la inoculación dual con Pisolithus tinctorius y Rhizophagus intraradices (Founoune et al., 2001; Misbahuzzaman y Newton, 2006), sin embargo, la información en torno al sistema Fraxinus uhdei-R. intraradices-P. tinctorius es casi inexistente, debido a que sólo se contaba con reportes de que las familias que forman asociación simbiótica tanto con hongos endomicorrízicos como con hongos ectomicorrízicos son: Caesalpiniaceae, Fagaceae, Juglandaceae, Myrtaceae, Salicaceae y Tiliaceae (Harley y Smith, 1983), por lo tanto este trabajo confirma lo reportado por Ambriz et al. (2010) en términos de la respuesta de la planta, pero en un sistema menos controlado. Desde luego no demuestra que la simbiosis simultánea ocurra de forma natural, pero sugiere que al menos una especie del género Fraxinus podría ser capaz de formar asociaciones con hongos ectomicorrízicos, lo que indica la necesidad de explorar esta posibilidad con individuos creciendo en condiciones naturales. Finalmente, debido al mejor desempeño de las plantas con inoculación dual, se podría sugerir que el uso de hongos micorrízicos (HMA y HEM) asociados a esta especie podría ser una herramienta para programas de restauración de suelos severamente degradados.

\section{Agradecimientos}

Se agradece el apoyo otorgado por la DGAPA de la UNAM a través del proyecto IN20212 y la beca PASPA para estancia sabática otorgada a RLC. ALBP agradece a beca doctoral otorgada por el CONACYT.

\section{Literatura citada}

Alexander I.J. y Fairley R.I. 1983. Effects of N fertilization on populations of fine roots and mycorrhizas in spruce humus. Plant and Soil 71:49-53.

Álvarez-Sanchez M.E., Hernández-Acosta E., Maldonado-Torres R. y Rivera-González M. 2013. Encalado y micorriza para corregir deficiencia de fósforo en un Andisol cultivado con Pinus halepensis. Madera y Bosques 19:7-16.

Ambriz E., Báez-Pérez A., Sánchez-Yáñez J.M., Moutoglis P. y Villegas J. 2010. Fraxinus-Glomus-Pisolithus symbiosis: Plant growth and soil aggregation effects. Pedobiologia 53:369-373.

Bocco G. y García-Oliva F. 1992. Researching gully erosion in Mexico. Journal of Soil and Water Conservation 47:365-367.
Callaway J.C., Zedler J.B. y Ross D.L. 1997. Using tidal salt marsh mesocosms to aid wetland restoration. Restoration Ecology 5:135-146.

Cañellas I., Finat L., Bachiller A. y Montero G. 1999. Comportamiento de planta de Pinus pinea en vivero y campo: ensayos de técnicas de cultivo de planta, fertilización y aplicación de herbicidas. Investigaciones Agrícolas: Producción y Protección Vegetal 8:335-359.

Crawley M.J. 2007. The R Book. John Wiley and Sons, Chichester.

Davel M., Tejera L., Honorato M. y Sepúlveda E. 2001. Plantación de pino ponderosa: Control de malezas, tipos de plantas y fertilizantes. Forestal 1:1-4.

Durán-Zuazo V.H. y Rodríguez-Pleguezuelo C.R. 2008. Soil-erosion and runoff prevention by plant covers. A review. Agronomy for Sustainable Development 28:65-86.

Duvert C., Gratiot N., Evrard O., Navratil O., Némery J., Prat C. y Esteves, M. 2010. Drivers of erosion and suspended sediment transport in three headwater catchments of the Mexican Central Highlands. Geomorphology 123:243-256.

Founoune H., Duponnois R., Bâ A. M., Sall S., Branget I., Lorquin J., Neyra M. y Chotte L.J. 2001. Mycorrhiza helper bacteria stimulate ectomycorrhizal symbiosis of Acacia holosericea with Pisolithus alba. New Phytologist 153:81-89.

Francis J.K. 1990. Fraxinus uhdei (Wenzig) Lingelsh. Fresno, tropical ash. Oleaceae. Olive family. USDA Forest Service, Southern Forest Experiment Station, Institute of Tropical Forestry, Washington, D.C.

Gómez-Romero M., Soto-Correa J.C., Blanco-García J.A., SáenzRomero C., Villegas J. y Lindig-Cisneros R. 2012. Estudio de especies de pino para restauración de sitios degradados. Agrociencia 46:795-807.

Harley J.L. y Smith S.E. 1983. Mycorrhizal Symbiosis. Academic Press, Londres.

Hayman D.S. 1983. The physiology of vesicular-arbuscular endomycorrhizal symbiosis. Canadian Journal of Botany 61:944963.

ISRIC [International Soil Reference and Information Centre] World Soil Information. 2013. <http://www.isric.org/isric/ webdocs/docs//major_soils_of_the_world/set6/ac/acrisol.pdf> (consultado: mayo, 2013).

Krüger M., Krüger C., Walker C., Stockinger H. y Schüßler A. 2012. Phylogenetic reference data for systematics and phylotaxonomy of arbuscular mycorrhizal fungi from phylum to species level. New Phytologist 193:970-984.

Maass J.M.M. y García-Oliva F. 1990. La conservación de suelos en zonas tropicales: el caso de México. Ciencia y Desarrollo 15:21-36.

Masera O., Ordoñez M.J. y Dirzo R. 1992. Emisiones de Carbono a partir de la deforestación en México. Ciencia 43:151- 153.

Méndez-Cortés H., Marmolejo-Monsiváis J.G., Cantú-Ayala C., Olalde-Portugal V., Estrada-Castillón E. y Posadas-Leal C. 2013. Respuesta de Cedrela odorata L. a diversos inoculantes micorrízicos procedentes de dos ecosistemas tropicales. Madera y Bosques 19:23-34.

Misbahuzzaman K. y Newton A. 2006. Effect of dual arbuscular ectomycorrhizal inoculation on mycorrhiza formation and growth in E. camaldulensis Dehnh. seedlings under different nutrient regimes. International Journal of Agriculture and Bio$\log y$ 8:848-854. 
Miller G.T. 1994. Ecología y Medio Ambiente: Introducción a la Ciencia Ambiental, el Desarrollo Sustentable y la Conciencia de Conservación del Planeta Tierra. Iberoamericana, México.

Monroy-Ata A., Estevez-Torres J., García-Sánchez R. y Ríos-Gómez R. 2007. Establecimiento de plantas mediante el uso de micorrizas y de islas de recursos en un matorral xerofilo deteriorado. Boletín de la Sociedad Botánica de México 80(suplemento):49-57.

Xiaojun N., Jianhui Z. y Zhengan S. 2013. Dynamics of soil organic carbon and microbial biomass carbon in relation to water erosion and tillage erosion. PLOS ONE 8:e64059.

Ocampo J.A. y Hayman D.S. 1981. Influence of plant interactions on vesicular-arbuscular mycorrhizal infection. II. Crop rotation and residual effects of non-host plants. New Phytologist 87:333-343.

Pérez-Moreno J. y Read D.J. 2004. Los hongos ectomicorrízicos, lazos vivientes que conectan y nutren a los árboles en la naturaleza. Interciencia 29:239-247.

Pimentel D. 2006. Soil erosion: a food and environmental threat. Environment, Development and Sustainability 8:119-137

Pohl M., Alig D., Kórner C. y Rixen C. 2009. Higher plant diversity enhances soil stability in disturbed alpine ecosystems. Plant and Soil 324:91-102.

Pringle A., Bever J.D., Gardes M., Parrent J.L., Rillig M.C. y Klironomos J.N. 2009. Mycorrhizal symbioses and plant invasions. Annual Review in Ecology Evolution and Systematics 40:699-715.

R Development Core Team. 2013. R: A language and environment for statistical computing. R Foundation for Statistical Computing, Vienna, <http://www.R-project.org>.

Rothstein D.E., Vitousek P.M. y Simmons B.L. 2004. An exotic tree alters decomposition and nutrient cycling in a Hawaiian montane forest. Ecosystems 7:805-814.

SEMARNAT [Secretaría de Medio Ambiente y Recursos Natura- les]. 1997. Secretaría de Medio Ambiente y Recursos Naturales. <http://app1.semarnat.gob.mx/dgeia/informe_resumen/03_ suelos/cap3.html > (consultado 11 de agosto de 2015)

Schüßler A. y Walker C. 2010. The Glomeromycota: A Species List With New Families and New Genera. The Royal Botanic Garden Edinburgh/The Royal Botanic Garden Kew/Botanische Staatssammlung Munich/Oregon State University, Gloucester.

Simard S.W. y Durall D.M. 2004. Mycorrhizal networks: a review of their extent, function, and importance. Canadian Journal of Botany 82:1140-1165.

Smith S.E., Facelli E., Pope S. y Smith F.A. 2010. Plant performance in stressful environments: interpreting new and established knowledge of the roles of arbuscular mycorrhizas. Plant Soil 326:3-20

Smith S.E. y Read D.J. 1997. Mycorrhizal Symbiosis. Academic Press, Londres.

Stabler L.B., Martin C.A. y Stutz J.C. 2001. Effect of urban expansion on arbuscular mycorrhizal fungal mediation of landscape tree growth. Journal of Arboriculture 27:193-202.

Szanser M., Kusińska A., Kisiel M. y Sieminiak D. 2011. Possibility of using diverse plant litter in soil restoration processes. Procedia Environmental Sciences 9:169-173.

Thomson B.D., Grove T.S., Malajczuk N. y Hardy G.E.St J. 1993. The effectiveness of ectomycorrhizal fungi in increasing the growth of Eucalyptus globulus Labill. in relation to root colonization and hyphal development in soil. New Phytologist 126:517-524.

Underwood A.J. 1997. Experiments in Ecology: Their Logical Design and Interpretation Using Analysis of Variance. Cambridge University Press, Cambridge.

Vierhelig H., Cougilan A.P., Wyss U. y Piche Y. 1998. Ink and vinagar, a simple staining technique for arbuscular mycorrhizal fungi. Applied and Environmental Microbiology 64:50045007.

Recibido: 2 de agosto de 2014

Aceptado: 15 de septiembre de 2014 